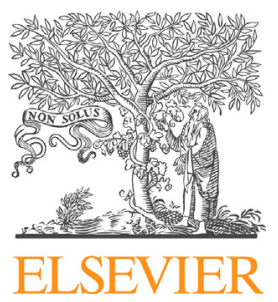

Urology Unit, Interdisciplinary Specialist Department, Meyer University Hospital, Viale Pieraccini 24, 50139, Firenze, Italy

Correspondence to: M. Lanciotti, Urology Unit, Interdisciplinary Specialist Department, Meyer University Hospital, Viale Pieraccini 24, 50139, Firenze, Italy

michele.lanciotti@unifi.it (M. Lanciotti)

Keywords

Proximal hypospadias; Urethroplasty; Bladder mucosal graft

Received 12 August 2016 Accepted 3 January 2017 Available online $x x x$

\title{
Proximal hypospadias repair with bladder mucosal graft: Our 10 years experience
}

\author{
Michele Lanciotti, Marta Betti, Antonio Elia, Luca Landi, \\ Maria Taverna, Chiara Cini, Lorenzo Masieri
}

\section{Summary}

Introduction and objective

A great variety of different surgical techniques has been described for proximal hypospadias repair and an ideal tissue has not been determined yet. We present our 10 years of surgical experience using a bladder mucosal graft for urethroplasty.

\section{Study design}

Bladder mucosal graft urethroplasty was performed between 2005 and 2015 in 50 patients with severe proximal hypospadias. The mean age of patients was 45.1 months (range 24-164 months). Hypospadias were perineal in 18 patients, scrotal in 22 , and penoscrotal in 10 . In all cases a chordee correction was performed and median time between the first and the second stages was 12 months (mean 17 months, range 4-68 months). Both foley catheter and a suprapubic cystostomy were positioned and maintained for 2-4 weeks. Follow-up was performed at $1-3$ and $6-12$ months after surgery, and afterwards annually with clinical examination and flowmetry test.

\section{Results}

Mean follow-up was 5.3 years (median 5, range $1-10$ years). Mean graft length was $57.4 \mathrm{~mm}$ (median $55 \mathrm{~mm}$, range
35-85 mm). Among all the only early complication registered was a postoperative infection in one patient $(2 \%)$ at the site of anastomosis. The long-term complications observed were urethrocutaneous fistula in nine patients $(18 \%)$, urethral stricture in 15 patients (30\%), meatal stricture in four patients $(8 \%)$, and prolapse of meatus in seven patients (14\%). The mean time of complication occurrence was 15 months (median 15.5 months, range 1-96 months). The functional and cosmetic appearance after surgery was satisfactory in $42 / 50$ patients ( $84 \%$ ) during the follow-up period.

\section{Discussion}

There is still an open debate regarding the optimal surgical approach for management of severe proximal hypospadias. Compared with other approaches, our technique showed acceptable results even though encumbered by slightly higher complication rates.

\section{Conclusion}

Our results show that bladder mucosal graft for primary severe proximal hypospadias in selected patients is a possible alternative to other commonly used techniques, with the aim of restoring recovery of the normal continuity of the distal urinary tract see figure below.

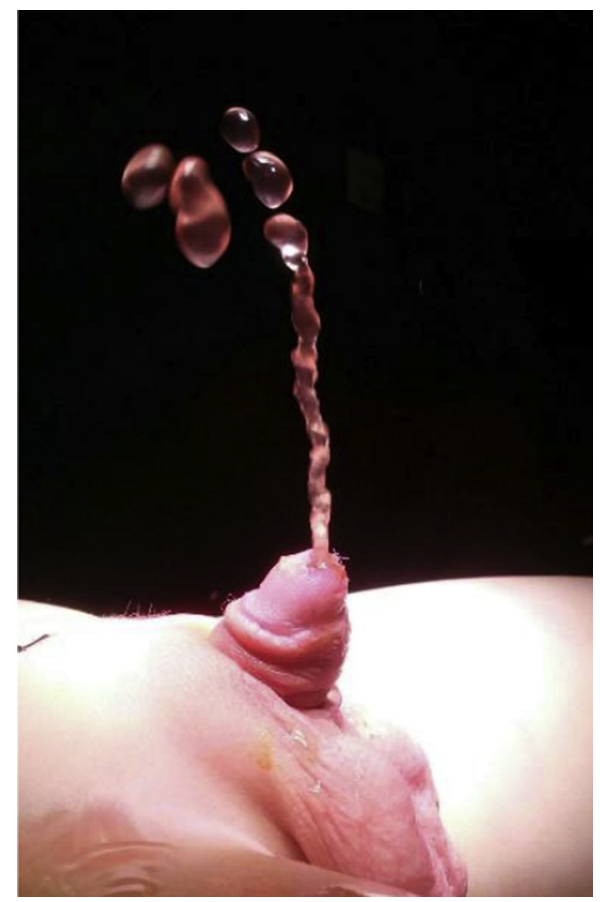

Figure Urethral micturition after bladder graft mucosa urethroplasty.

http://dx.doi.org/10.1016/j.jpurol.2017.01.011

1477-5131/@ 2017 Journal of Pediatric Urology Company. Published by Elsevier Ltd. All rights reserved. 


\section{Introduction}

Hypospadias is the most common congenital malformation of the penis, caused by hypoplasia of the ventral tissues in varying degrees [1-6]. Therapeutic treatment is surgical, with the purpose of reconstructing a straight penile shaft with the external urethral meatus as close as possible to its normal position, to allow urination in standing position and, as an adult, a normal penetration. Among all hypospadias, proximal ones are less than $20 \%$ [7], and complex urethral reconstruction is one of the most challenging issues in pediatric urology, typically requiring various reconstructive surgeries [8]. The multiplicity of surgical techniques for proximal hypospadias with chordee reflects the struggle to decrease the rate of serious complications after repair, with data reported in the literature showing a complication rate between $14 \%$ and $61 \%$ [9]. Continuous advances in surgical treatment mean there are several potential reconstruction techniques for proximal hypospadias and many tissues have been used as urethral graft substitutes when local penile or preputial skin is deficient or unavailable. The most commonly used free grafts to substitute long urethral defects include buccal mucosa, de-epithelialized or nonhair bearing skin, posterior auricular skin, and bladder mucosa [9]. Mucosal grafts are readily available and make successful urethral substitutes according to surgeon preferences. Memmelaar [10] was the first to use a different mucosal substitute and perform bladder mucosa-free graft urethroplasty, in particular in treatment of complex and/or secondary cases, using single or staged repair as appropriate $[11,12]$. Debate on correction of proximal cases permeates the literature with divergent views on the role of plate transection and/or augmentation with grafts, because of the various short- and medium-term results reported [8].

We present our experience in urethroplasty with tubularized bladder mucosal graft in a significant series of children with initial severe proximal hypospadias. We describe the incidence of complications, including fistula, stenosis, and meatal mucosal prolapse during a long followup period.

\section{Materials and methods}

From January 2005 to February 2015, 50 male patients with severe proximal hypospadias (Fig. 1) underwent urethral reconstruction using autologous free bladder mucosal graft at the Meyer University Hospital of Florence, Italy. Three different surgeons performed this technique during these 10 years.

The meatus was perineal in 18 (36\%) patients, scrotal in 22 (44\%), and penoscrotal in $10(20 \%)$ patients. All patients were assessed for current voiding status, cosmesis, and complications, and in all patients abdomen ultrasound, genetic and endocrinological counseling were routinely performed in the preoperative period. This technique was indicated exclusively for primary proximal hypospadias, accurately selected, to ensure the continuity of the urothelial path.

The frequent association of the ventral curvature of the penis to proximal hypospadias mean that a two-stage repair was routinely performed. The two-stage approach has the advantage of dealing with corrected tissues with an already

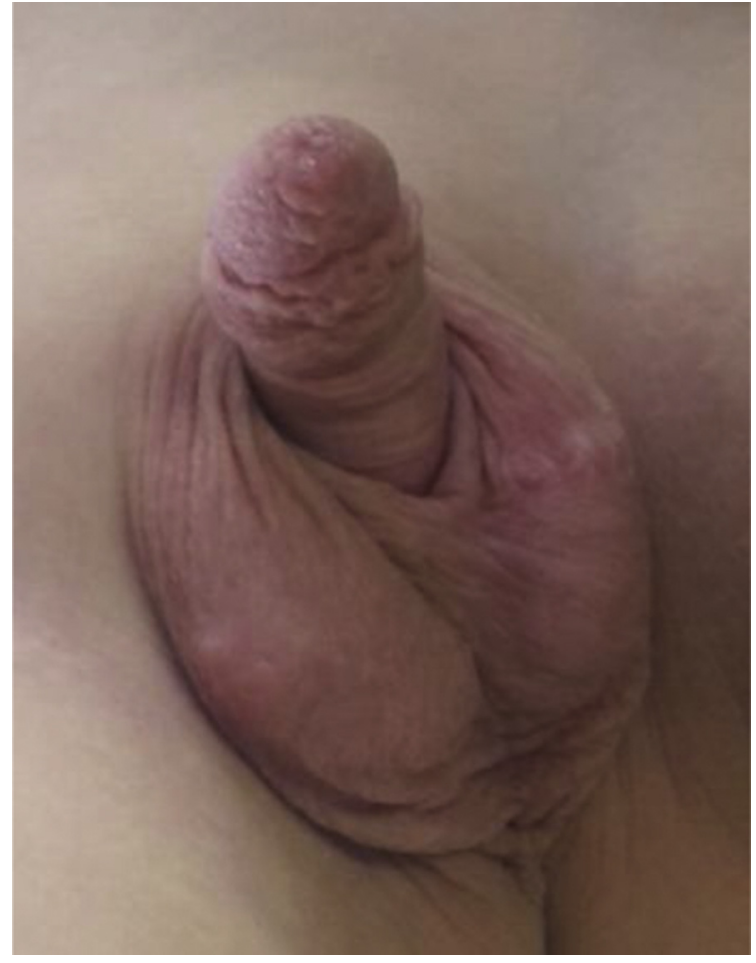

Figure 1 A posterior hypospadias.

mature vascularized tissue from which we consequently could fashion the neo-urethra, to obtain functional refinement aside from potentially good cosmetic results. At the first stage, chordee tissue was removed in addition to skin detethering, Nesbit wedges, and Byars reconstruction to straighten the penis [13]. In addition to penile shaft malformations, other genitourinary malformations were detected. Specifically, we found that 18 children had undescended testicle, three cases presented posterior urethral valves, vesicoureteral reflux in two patients, and in one case there was associated unilateral renal agenesis.

We preferred a two-stage procedure after straightening curvature, and carrying out urethroplasty when a correct scarring occurred on the penile shaft $[14,15]$. At the second stage, the bladder mucosal graft urethroplasty was performed as already described in the literature [16,17]. After a small Pfannenstiel incision, the bladder was distended through the bladder catheter so that its anterior surface was identified easily. An incision in the detrusor muscle was carried deeply until the mucosa was exposed to obtain the proper graft. Usually the graft was $10 \%$ longer and $20 \%$ wider than needed [18], and our mean bladder mucosal graft length was $57.4 \mathrm{~mm}$, median $55 \mathrm{~mm}$, ranging from $35 \mathrm{~mm}$ to $85 \mathrm{~mm}$ (Fig. 2A). While the bladder and suprapubic incisions were closed and a suprapubic tube was left indwelling, the free graft of bladder mucosa was prepared surrounding a $10 \mathrm{CH}$ [14] multiple perforated silicone rubber tube to obtain the mucosal surface directed to an inward fashion (Fig. 2B). The graft was kept continuously wet with saline solution until it was ready for transfer [16].

The hypospadiac meatus was freed up with a circumferential incision that was carried deeply for at least $85 \mathrm{~mm}$. Tunneling for the tubularized bladder mucosal graft was initiated at the new glandular meatus and carried 

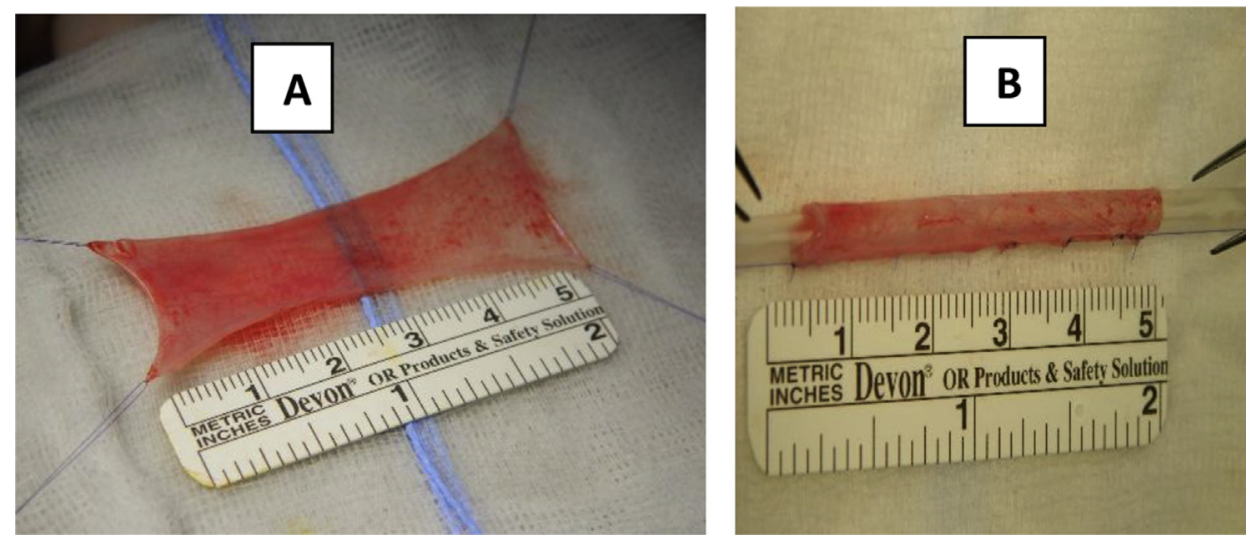

Figure 2 Bladder mucosa graft (A) and after tubularization on $10 \mathrm{CH}$ silicone tube (B).

deeply using scissors and dilatators, within the subcutaneous tissue of the ventral surface of the penis to the proximal hypospadiac meatus, where the true urethra had been dissected and spatulated before. The bladder mucosal graft was subsequently drawn into the tunnel until the tip of the glans penis, to preserve overlying dartos supporting layers in the proximal side and to ensure that the urethra was at an adequate depth and not adherent to the ventral skin. Then, we performed an in-situ anasthomosis to the urethral plate widely spatulated, with a running suture of absorbable $6 / 0$ suture (Fig. 3 ). Closure of the subcutaneous tissues was performed in double layer with $6 / 0$ suture to eliminate dead space. The new meatus was located in the proper position at the glans apex (Figs. 4 and 5).

A compressive dressing was applied for 2 days. The urinary catheter was maintained to dwelling for 2 weeks. This was necessary to verify the proper capacity and to analyze micturition and verify correct healing. The suprapubic cystostomy was removed 2 weeks later, ambulatory by verifying micturition. Surgical complications and additional urethral surgeries following definitive hypospadias repair were defined as procedures that required surgical intervention under general anesthesia (Grade IIIb Clavien classification). We adopted the Hypospadias Objective Penile Evaluation (HOPE)-scoring system to assess the postoperative results during the follow-up period.

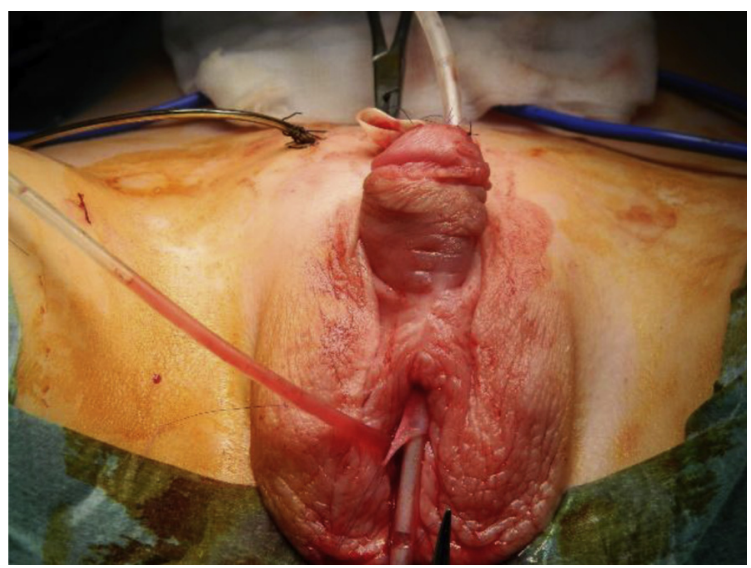

Figure 3 Bladder mucosa graft tunneled from proximal hypospadiac meatus and tip of the glans.

\section{Results}

Mean follow-up was 5.3 years, median 5 years, with a range of 1-10 years and no patient was lost during the follow-up period. Clinical evaluation of the urine stream, flowmetry, the cosmetic appearance, and persistent curvature in erection were routinely performed at $1-3$ and $6-12$ months postoperatively and then annually until puberty during follow-up. Urethral calibration to detect stenosis was never performed on a systematic basis. Mean patient age at chordee correction was 24 months (median 18 months, range $10-150$ months), and we performed urethroplasty with bladder mucosal graft after 17 months (median 12, range 4-68 months), in patients with mean age 42 months (median 38 months, range 23-164 months), as reported in Table 1.

The functional and cosmetic appearance after surgery was satisfactory in $42 / 50$ patients $(84 \%)$ as measured by the HOPE scoring system during the follow-up period.

The mean time of complication occurrence was 15 months (median 15.5 months, range 1-96 months). The

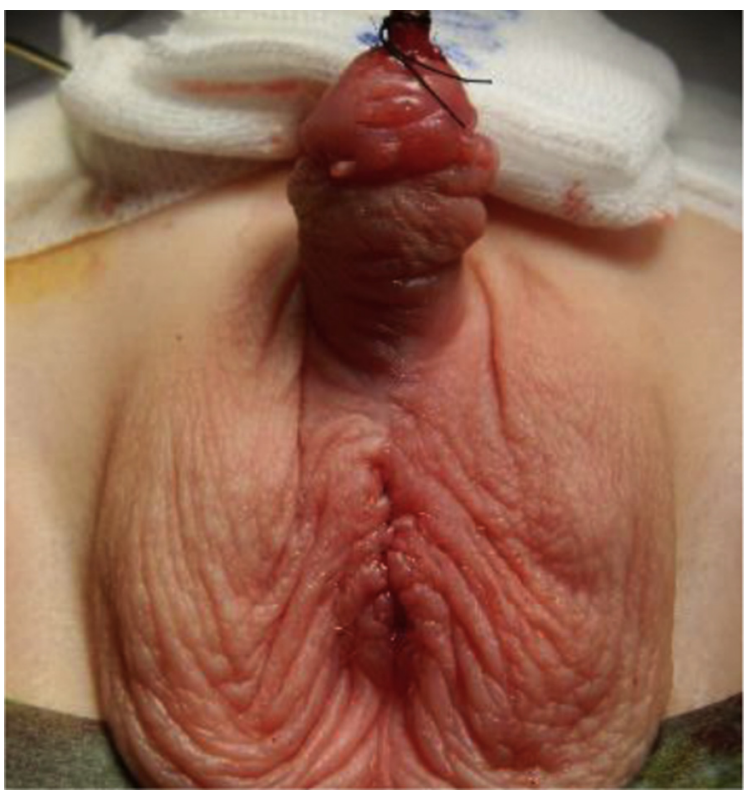

Figure 4 Final appearance. 


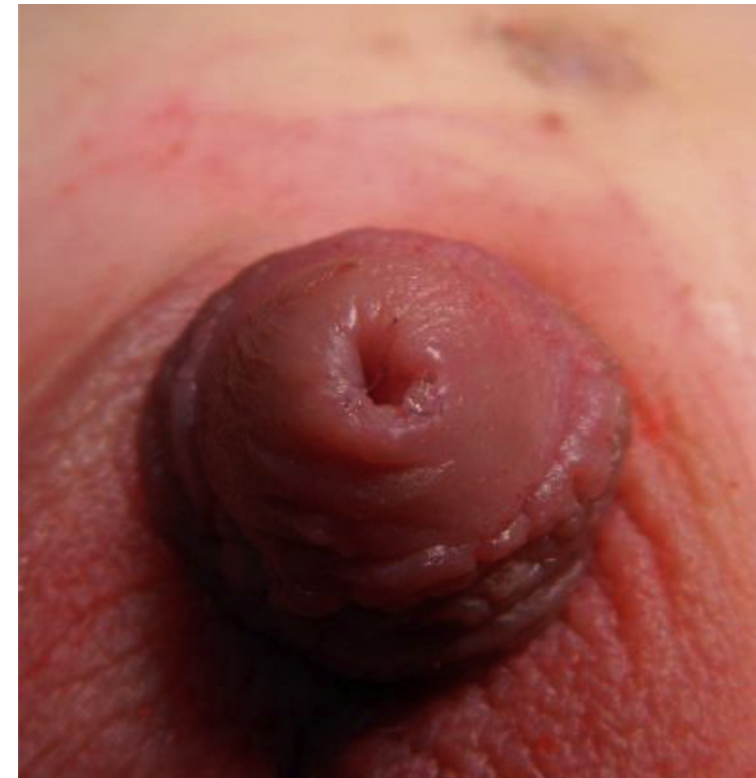

Figure 5 Final appearance of neo-urethral meatus.

early complication registered during hospitalization was a postoperative infection in one patient ( $2 \%, 1 / 50$ patients) at the site of anastomosis between the bladder mucosal graft and native urethra, provocating a urethrocutaneous fistula.

Medium-to long-term complications (range 1-96 months) were anasthomosis stricture in 15 patients (30\%), and $10 / 15(66.6 \%)$ occurred in the first 5 years of practice. Urethrocutaneous fistula at the anastomosis site occurred in nine patients $(18 \%)$, urethral diverticula in six patients (12\%), meatal stricture in four patients $(8 \%)$, recurring curvature in five patients $(10 \%)$, and prolapse of meatus in seven patients $(14 \%)$. Fifteen patients $(30 \%)$ presented with multiple complications, as reported in Table 2 , resulting in an overall complication rate of $46 \%$.

In 13 patients with urethral and meatal stricture only Hegar dilatation was performed, whereas in six patients a further meathotomy was necessary. Urethrocutaneous fistulas were further corrected after 6-9 months. No bladder or abdominal complications occurred after surgery. In three

Table 1 Patients' characteristics.

\begin{tabular}{ll}
\hline Site of hypospadias & 50 patients: $n(\%)$ \\
\hline Perineal & $18(36)$ \\
Scrotal & $22(44)$ \\
Penoscrotal & $10(20)$ \\
Surgical aspects & Mean (median, range) \\
Patient age at chordee & $24(18,10-150)$ \\
$\quad$ correction, months & $17(12,4-68)$ \\
Expectation period between & \\
two surgical steps, months & \\
$\begin{array}{l}\text { Patient age at urethroplasty, } \\
\text { months }\end{array}$ & $42(38,23-164)$ \\
$\quad \begin{array}{l}\text { Follow-up, years } \\
\text { Time of complication } \\
\text { occurrence }\end{array}$ & $5.3(5,1-10)$ \\
\hline
\end{tabular}

Table 2 Complication reported after surgery.

\begin{tabular}{ll}
\hline Type of complication & 50 patients: $n(\%)$ \\
\hline Overall complication & $23(46)$ \\
Anasthomosis stricture & $15(30)$ \\
Urethrocutaneous fistula & $9(18)$ \\
Urethral diverticula & $6(12)$ \\
Meatal stricture & $4(8)$ \\
Recurrent curvature & $5(10)$ \\
Prolapse of meatus & $7(14)$ \\
Patients with multiple complication & $15(30)$ \\
\hline
\end{tabular}

patients a further urethroplasty was necessary, because of urethral diverticulum in two patients and urethrocutaneous fistula in one patient.

During the follow-up period, even in patients who underwent surgical repair and correction of each complication, no voiding alteration was registered on flowmetry testing.

\section{Discussion}

Proximal hypospadias with chordee is one of the most challenging issues faced by pediatric urologists, and typically requires multiple reconstructive surgeries. Among several potential treatment techniques, use of oral mucosa has proved successful thanks to its easier and less invasive approach [9]. Bladder mucosal graft has several theoretical advantages, it is not hair-bearing, tends to re-epithelialize exposed surfaces, and has great distensibility [19]. The bladder mucosa is an attractive material for reconstruction of the neourethra in repeat repair of hypospadias and other complex urethral anomalies, because it is accustomed to urine exposure, resistant to shrinkage, and in plentiful supply [20]. The use of bladder mucosa as a free graft was first reported by Memmelaar [10] in 1947, then revived by Hendren $[16,17]$ and Ransley [21]. Since then, bladder mucosal graft has been considered a readily available and successful urethral substitute. It has gained some interest although use has remained limited, particularly because of complicated cases when local penile or preputial skin are not available $[10,16,21,22]$. The first objective of the technique was to solve the functional problem and simultaneously create a pleasant final appearance, while minimizing the risk of fistula or stenosis as second objective. Urethrocutaneous fistulas were further corrected after 6-9 months of expectation period according to the literature [23].

At our tertiary referral centre, the technique using an autologous bladder mucosal graft for urethroplasty was used in properly selected patients as first choice for certain severe initial proximal hypospadias correction as suggested by Memmelaar and Hendren [10,16]. Other authors reported this technique in only selected cases of patients, who previously underwent urethroplasty for proximal hypospadias with further complication, and when other techniques were not available, such as preputial pedicle tissue flaps [12,18,23-25].

The approach we performed should not be considered a replacement to the already widely adopted techniques. We think it offers an alternative to these techniques in 
selected cases, such as for extensive urethral salvage in hypospadias where the preferred easier graft options have already been used up, or in patients with extensive Lichen Sclerosus et Atrophicus of the urethra.

The great majority (84\%) of our patients achieved successful cosmetic and functional outcome following this procedure, as measured by the HOPE-score during followup, even if it was necessary to repair and correct several complications. Overall complication rates reported in the literature range from $14 \%$ to $60 \%[9,21,25,26]$, thus our complication rate is broadly comparable, even if the anasthomosis stricture was considerably higher. Nevertheless, patients included in this study had severe proximal hypospadias, and any problem requiring treatment was attended to during the extremely long follow-up. The majority of our patients required only minor procedures, such as one or more dilatations under local anesthetic, with few requiring more complex surgical resolution in the operating room.

As reported in the literature, early problems with meatal prolapse and stricture of bladder mucosal graft were believed to result from exposure of the graft to the air, with stickiness and hypertrophy from columnar metaplasia and development of mucin secreting glands $[21,25]$. The meatal problems in this study comprised meatal stricture in four patients $(8 \%)$ and prolapse of meatus in seven patients $(14 \%)$, lower than rates reported in the literature, ranging from $24 \%$ to $100 \%$ [18,20-22,25]. These problems probably occur because the exposed mucosa behaves similarly to extrophied bladder mucosa, being hypertrophic and sticky, and eventually metaplastic leading to prolapse and/or stenosis [21]. Thus to reduce meatal protrusion, the tunnel we performed was not extensive and the mucosa was carefully stretched to avoid redundancy at the tip. The suture on the glans was inverted to reduce air contact and to avoid prolapse.

Aside from meatal problems, most of the complications following bladder mucosa-graft urethroplasty were similar to those encountered with other grafts, and were managed accordingly.

Despite attempts to prevent stenosis and possible diverticula formation by careful preparation of the glans channel with excision of a generous button of glans tissue, stenosis continues to be a problem although a minor one. The depth of the tunnel and the ability to perform anastomosis at this deep level were fundamental to prevent formation of fistula and stenosis. Our fistula rate of $18 \%$ is in accordance with the literature, and not excessive in this challenging group of patients $[21,26]$. Thus, resection of deep intraglandular tissue may be necessary to ensure an adequate channel [14]. Even closely following such processes, we report a urethral stricture in 15 patients $(30 \%)$ that is higher than the $14 \%$ rate reported in literature [26]. Substantially $10 / 15(66.6 \%)$ occurred in the first 5 years of practice versus $5 / 15(33.4 \%)$ in the last 5 years, perhaps because of continuous improving surgical experience and development of the surgical technique. Better lack of tension, wide spatulation, and small suture material are recommended to prevent strictures. Moreover, we noted a higher stricture rate when the graft length was longer, although not statistically significant, particularly over a median value of $55 \mathrm{~mm}$. Unfortunately, as our patients presented with severe proximal hypospadias, it was not possible to reduce the graft length.

The first limitation of the study is not having compared our technique with the buccal mucosa technique. Second, our large series of operations was performed by three different surgeons at our hospital. The third limitation is in the follow-up, in which urethral dilatation was not routinely performed, so it was not always possible to prevent stenosis.

The aim of our work was to perform a critical view of a large series of patients. We believe that this will stimulate scientific discussion and constructive debate, with the purpose of making important further comparison among different techniques. Debate continues regarding the optimal surgical approach for management of severe proximal hypospadias, and our technique could be considered a possible alternative to other widely accepted approaches in selected patients, although further comparisons are necessary.

\section{Conclusion}

Considering the difficult and complex nature of this challenging patient cohort, the complications encountered are within an acceptable range, with satisfactory cosmetic results. Thus, the bladder mucosal graft represents a possible alternative for selected patients with severe proximal hypospadias. The aim of our paper was to allow for further constructive debate on this technique, although other widely adopted techniques have slightly superior warranties especially as the primary approach.

\section{Conflict of interest}

None.

\section{Funding}

None.

\section{References}

[1] Stokowski LA. Hypospadias in the neonate. Adv Neonatal Care 2004;4(4):206-15.

[2] Baskin LS, Himes K, Colborn T. Hypospadias and endocrine disruption: is there a connection? Environ Health Perspect 2001;109(11):1175-83.

[3] Wein AJ, Kavoussi LR, Novick AC, Partin AW, Peters CA. Campbell-Walsh Urology Review. 3rd ed. Saunders Elsevier; 2007.

[4] Bauer SB, Retik AB, Colodny AH. Genetic aspects of hypospadias. Urol Clin N Am 1981;8:559.

[5] Springer A, van den Heijkant M, Baumann S. Worldwide prevalence of hypospadias. J Pediatr Urol 2016;12(3). 152.e1-e7.

[6] Marrocco G, Vallasciani S, Fiocca G, Calisti A. Hypospadias surgery: a 10-year review. Pediatr Surg Int 2004;20(3):200-3.

[7] Demirbilek S, Kanmaz T, Aydin G, Yücesan S. Outcomes of onestage techniques for proximal hypospadias repair. Urology 2001;58:267-70.

[8] Pippi Salle JL, Sayed S, Salle A, Bagli D, Farhat W, Koyle M, et al. Proximal hypospadias: a persistent challenge. Single 
institution outcome analysis of three surgical techniques over a 10-year period. J Pediatr Urol 2016;12(1). 28.e1-e7.

[9] Fine R, Reda EF, Zelkovic P, Gitlin J, Freyle J, Franco I, et al. Tunneled buccal mucosa tube grafts for repair of proximal hypospadias. J Urol 2015;193:1813-8.

[10] Memmelaar J. Use of bladder mucosa in one stage repair of hypospadias. J Urol 1947;58:68-73.

[11] Hadidi AT, Azmy AF. Hypospadias Surgery, an illustrated guide. Berlin Heidelberg: Springer-Verlag; 2004.

[12] Faure A, Bouty A, Nyo YL, O’Brien M, Heloury Y. Two-stage graft urethroplasty for proximal and complicated hypospadias in children: a retrospective study. J Pediatr Urol 2016;12(5). 286.e1-e7.

[13] Byars LT. A technique for consistently satisfactory repair of hypospadias. Surg Gynecol Obstet 1955;100(2):184-90.

[14] Ehrlich RM, Reda EF, Koyle MA, Kogan SJ, Levitt SB. Complications of bladder mucosal graft. J Urol 1989;142(2 Pt 2): 626-7. discussion 631.

[15] Fu Q, Deng CL. Ten-year experience with composite bladder mucosa skin grafts in hypospadias repair. Pediatr Urol 2006; 67(6):1274-7.

[16] Hendren H, Reda EF. Bladder mucosa graft for construction of male urethra. J Pediatr Surg 1986;21(3):189-92.

[17] Hendren WH, Crooks KK. Tubed free skin graft for construction of male urethra. J Urol 1980;123:858.
[18] Mollard P, Mouriquand P, Bringeon G, Bugmann P. Repair of hypospadias using a bladder mucosal graft in 76 cases. J Urol 1989;142:1548-50.

[19] Coleman JW. The bladder mucosal graft technique of hypospadias repair. J Urol 1981;125:708-10.

[20] Kinkead TM, Borzi PA, Duffy PG, Ransley PG. Long-term followup of bladder mucosa graft for male urethral reconstruction. J Urol 1994;151:1066-8.

[21] Ransley PG, Duffy PG, Oesch IL, Van Oyen P, Hoover D. The use of bladder mucosa and combined bladder mucosa/preputial skin grafts for urethral reconstruction. J Urol 1987;138: 1096.

[22] Ozgök Y, Ozgür Tan M, Kilciler M, Tahmaz L, Erduran D. Use of bladder mucosal graft for urethral reconstruction. J Urol 2000; 7:355-60.

[23] Li LC, Zhang X, Zhou SW, Zhou XC, Yang WM, Zhang YS. Experience with repair of hypospadias using bladder mucosa in adolescents and adults. J Urol 1995;153:117-9.

[24] Koyle MA, Ehrlich RM. The bladder mucosal graft for urethral reconstruction. J Urol 1987;138:1093-5.

[25] Keating MA, Cartwright PC, Duckett JW. Bladder mucosa in urethral reconstructions. J Urol 1990;144:827-34.

[26] Rober PE, Perlmutter AD, Reitelman C. Experience with 81, 1stage hypospadias/chordee repairs with free graft urethroplasties. J Urol 1990;144(2 Pt 2):526-9. discussion 530. 